Check for updates

Cite this: RSC Adv., 2019, 9, 13658

Received 18th March 2019

Accepted 27th April 2019

DOI: 10.1039/c9ra02087j

rsc.li/rsc-advances

\section{Laser wavelength modulated pulsed laser ablation for selective and efficient production of graphene quantum dots $\uparrow$}

\author{
Sukhyun Kang, $\$^{\mathrm{ab}}$ Jeong Ho Ryu, $\$^{\mathrm{c}}$ Byoungsoo Lee, ${ }^{\mathrm{a}}$ Kyung Hwan Jung, ${ }^{\mathrm{a}}$ \\ Kwang Bo Shim, ${ }^{b}$ Hyuksu Han (D) *ad and Kang Min Kim*a
}

\begin{abstract}
Graphene quantum dots (GQDs) and graphene oxide quantum dots (GOQDs) can be used in different applications such as optoelectronic and biomedical applications, respectively. Hence, the selective synthesis of GQDs and GOQDs is highly desirable but challenging. Here, we present GQDs and GOQDs selectively prepared by an easy and simple pulsed laser ablation in liquid (PLAL) method by controlling the laser wavelength. The obtained GQDs and GOQDs showed a significantly different optoelectronic nature mainly due to the existence of surface oxygen-rich functional groups (e.g. carboxyl or hydroxy groups). Also, we described a possible mechanism for the formation of oxygen functional groups during the PLAL process based on the Coulomb explosion model, which can give further insight for designing functional carbon materials.
\end{abstract}

Graphene quantum dots (GQDs) and graphene oxide quantum dots (GOQDs) are zero-dimensional graphene nanomaterials composed of one or few-layer graphene sheets. ${ }^{1,2}$ Compared to large size graphene, GQDs or GOQDs exhibit unique optoelectrical properties due to quantum confinement or edge effects related to oxygen-rich functional groups..$^{3-6}$ Especially, GQDs exhibit a high quantum yield with excellent photostability owing to the intrinsic band structure and physicochemical robustness, which is beneficial for optoelectronic applications. ${ }^{7-10}$ In addition, although the optical properties of GOQDs are less fascinating compared to those of GQDs, GOQDs have a high potential for biomedical applications, such as drug delivery systems (DDSs) and bioimaging, thanks to excellent biocompatibility with acceptable optical efficiency. ${ }^{11-14}$

To date, GQDs and GOQDs have been prepared mainly via wet chemical cutting routes. However, chemical reactions in strong acid as well as long-term washing procedure are generally required during a wet chemical process, which limits the practical applications of GQDs and GOQDs. ${ }^{15-19}$ As an alternative method, pulsed laser ablation in liquid (PLAL) has recently

${ }^{a}$ Korea Institute of Industrial Technology, Gwahakdanji-ro 137-41, Gangwon-do, 25440, Republic of Korea.E-mail:kmkim@kitech.re.kr

${ }^{b}$ Department of Materials Science and Engineering, Hanyang University, 17 Haengdang-dong, Seongdong-gu, Seoul 133-791, Republic of Korea

'Department of Materials Science and Engineering, Korea National University of Transportation, 50 Daehak-ro, Chungju-si, Chungbuk 380-702, Republic of Korea

${ }^{d}$ Department of Materials Science and Engineering, Hongik University, Sejong-ro 2639, Sejong, Republic of Korea. E-mail: hhan@hongik.ac.kr

† Electronic supplementary information (ESI) available. See DOI: 10.1039/c9ra02087j

\$ These two authors contributed equally to this work. attracted great attention for the synthesis of GQDs and GOQDs. The PLAL method is facile, simple, and environmentally benign because it does not require strong acidic chemical and postpurification steps. ${ }^{20-23}$

Coulomb explosion model has been proposed to account for the ablation mechanism of carbon materials by PLAL. ${ }^{21,22}$ Briefly, when the laser pulses inject into the carbon precursor, ionization occurs by multiphoton absorption, resulting in the formation of high temperature and pressure plasma plume. In the plasma plume, Coulomb explosion can take place and subsequently the carbon precursors are ablated to the quantum size leading to the formation of GQDs. Simultaneously, the Coulomb explosion can decompose the solvent during the formation of GQDs or GOQDs. ${ }^{23,24}$ The decomposed small molecules in the solvent can functionalize the GQDs by inducing chemical bonds with the surface of the GQDs. This can significantly alter the final optoelectronic properties of GQDs. Importantly, laser wavelength has a strong relationship with the decomposition of the solvent given that reaction area and depth between light and liquid matter is mainly determined by the wavelength of laser. Hence, not only natures of solvent but also the laser wavelength can significantly influence the optoelectronic functionalities of GQDs prepared by PLAL method. In our previous report, the effects of chemical properties of solvent on the functionalization of GQDs prepared by PLAL has been studied..$^{25}$ However, to our best knowledge, relationships between laser wavelength and the functionalization of GQDs during PLAL still remain as unexplored scientific area.

GQDs and GOQDs were fabricated by PLAL methods using MWCNTs as carbon source in high-purity ethanol. Briefly, $50 \mathrm{mg}$ of MWCNTs was dispersed in $500 \mathrm{~mL}$ of ethanol by 
ultrasonication. A Q-switch ND:YAG laser system was employed for PLAL. The MWCNTs suspension, which forms a vertical water column, was ablated by using the horizontal pulsed laser with the wavelength of $355 \mathrm{~nm}$ and $532 \mathrm{~nm}$, respectively, at a repetition rate of $10 \mathrm{~Hz}$ and ablation energy of $50 \mathrm{~mJ}$. The pulsed laser beam was focused on the center of MWCNTs suspension (see the ESI† for Experimental details) (Fig. 1).

The morphology of starting MWCNTs was characterized by transmission electron microscopy (TEM) (Fig. S1†). The TEM images of MWCNTs shows that the wall of CNT has consisted of approximately 25-27 layers of graphene sheets (Fig. S1†). An average diameter of the tube was about $25 \mathrm{~nm}$. Ultrathin morphology of MWCNTs can effectively suppress the pyrolytic phenomenon during PLAL, which is desirable for the formation of homogeneous GQDs or GOQDs colloidal. ${ }^{25}$ The MWCNTs was transformed to GQDs by PLAL using $532 \mathrm{~nm}$ laser source. GQDs

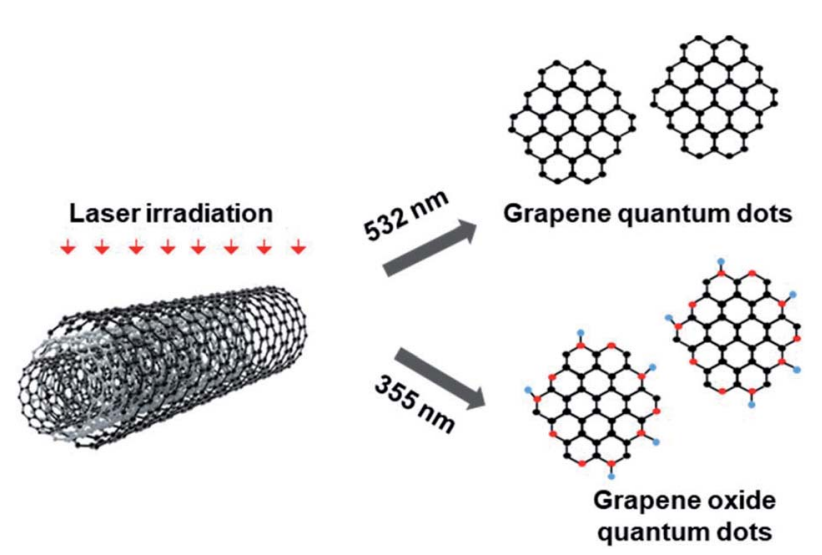

Fig. 1 Representative schematic for the possible mechanism of the transform MWCNTs to GQDs and GOQDs using PLAL process (oxygen-rich site are shown as red dots). with size less than $5 \mathrm{~nm}$ was obtained after $10 \mathrm{~min}$ of ablation (Fig. 2a). The size distribution of GQDs is fitted by Gaussian curves and shown in Fig. S2a. $\dagger$ An average diameter of 1-5 nm was calculated by counting more than 30 numbers of GQDs. HR-TEM image and corresponding fast Fourier transform (FFT) pattern of GQDs show a highly crystalline structure with a lattice parameter of $\sim 0.24 \mathrm{~nm}$ (Fig. 2b). No crystalline features that correspond to graphite, such as [002] plane, were observed by HR-TEM. ${ }^{26,27}$ The atomic force microscopy (AFM) data in Fig. 2c and $d$ illustrate the topographic morphology and the height distribution of GQDs. The height line profile in Fig. 2d shows that the thickness of GQDs is less than $1.5 \mathrm{~nm}$ corresponding to 3-4 graphene layers. Statistical analysis reveals that more than $85 \%$ of the GQDs has a thickness between 0.5 and $1.5 \mathrm{~nm}$, indicating that the produced GQDs has a mono or a few layered structures.

To investigate the effect of laser wavelength on the functionalities of GQDs, we employed the $355 \mathrm{~nm}$ laser source (3.4 eV) for PLAL process, which has much higher photon energy than $532 \mathrm{~nm}$ laser source $(2.33 \mathrm{eV})$. MWCNTs was ablated in ethanol using the laser source with a wavelength of $355 \mathrm{~nm}$. All the experimental parameters except for laser wavelength were kept identical during PLAL. X-ray photoelectron spectroscopy (XPS) was firstly carried out for the compositional analysis of GQDs ablated by different laser wavelengths, 532 and $355 \mathrm{~nm}$. For the comparison purpose, starting MWCNTs was also studied by XPS. XPS spectra of C 1s for MWCNTs only presents $\mathrm{sp}^{2}$ carbon peak at the binding energy of $284.4 \mathrm{eV}$ (Fig. S3 $\dagger$ ). Similarly, the GQDs ablated by $532 \mathrm{~nm}$ shows a major peak at $284.4 \mathrm{eV}$, which can be attributed to $\mathrm{sp}^{2}$ carbon peak. The deconvoluted peaks at $286.0 \mathrm{eV}$ and $288.8 \mathrm{eV}$ are associated with hydroxyl or carboxyl functional groups bonded in $\mathrm{sp}^{3}$ orbital structure, respectively (Fig. 3a). According to quantitative (a)

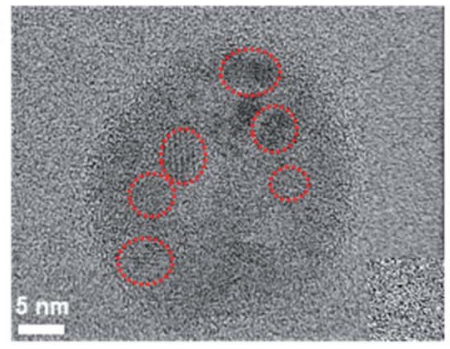

(b)
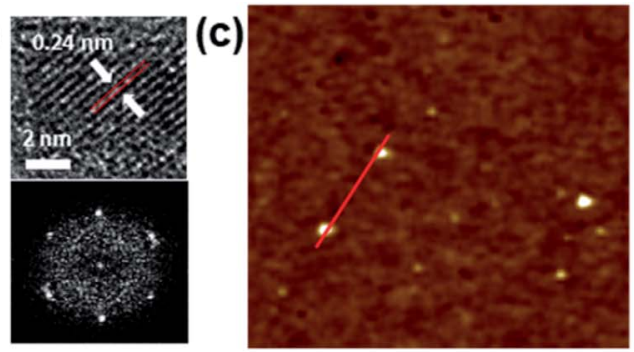

(e)

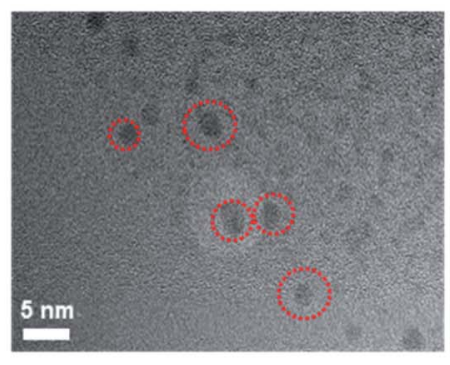

(f)

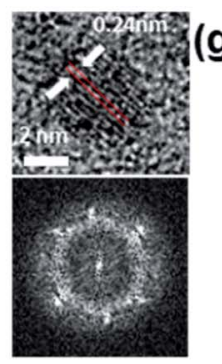

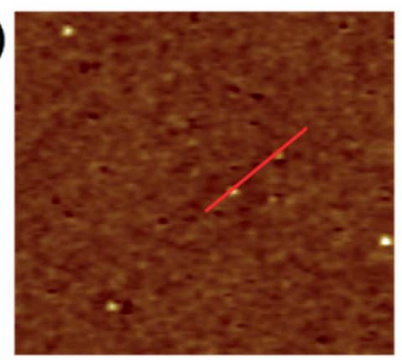

(d)

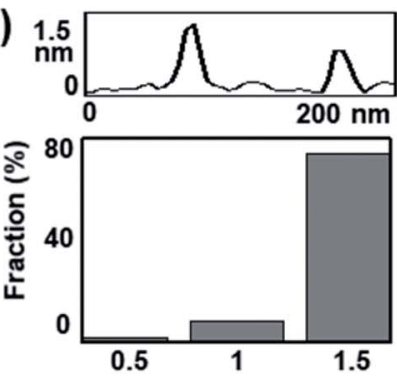

(h)
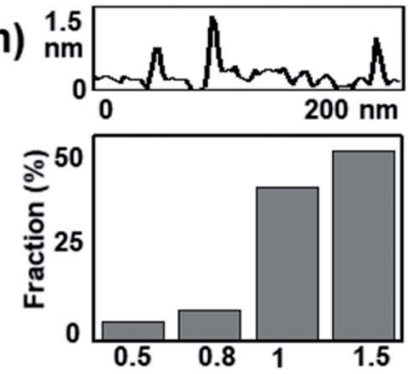

Fig. 2 Morphology characterization of GQDs and GOQDs. (a, b) TEM and HR-TEM images of GQDs and corresponding FFT image. (c, d) AFM image and height profile, height distribution of GQDs, respectively. (e, f) TEM and HR-TEM images of GOQDs and corresponding FFT images. ( $g$, h) AFM image and height profile, height distribution of GOQDs, respectively. 
analysis of the XPS spectra, fractions of $\mathrm{sp}^{2}$ and $\mathrm{sp}^{3}$ carbon peaks are estimated as $86.55 \%$ and $13.45 \%$, respectively. In contrast, the GOQDs ablated by $355 \mathrm{~nm}$ show a significantly increased fraction of $\mathrm{sp}^{3}$ carbon peaks from oxygen-rich functional groups (Fig. 3b). Quantitative fractions are calculated as $55.91 \%$ and $44.09 \%$ for $\mathrm{sp}^{2}$ and $\mathrm{sp}^{3}$ carbon peaks, respectively (Table S1 $\dagger$ ), indicating that GOQDs ablated by shorter wavelength $(355 \mathrm{~nm})$ is literally GOQDs rather than GQDs. These results suggest that the surface functionalization of GQDs by oxygeneous species can be easily achieved by simply changing laser wavelength for PLAL process.

TEM images revealed that the GOQDs have a diameter less than $5 \mathrm{~nm}$ (Fig. 2e). The HR-TEM image and the corresponding FFT pattern showed that the crystal structure of the GOQDs has a similar lattice structure with that of GQDs (Fig. 2b). The size distribution of GOQDs was calculated by counting about 45 GOQDs, and the result was fitted by Gaussian curves (Fig. S2b $\dagger$ ). An average diameter of 1-5 $\mathrm{nm}$ was obtained for the GOQDs. In addition, the height profile for GOQDs was obtained by using AFM, indicating that more than $90 \%$ of the GOQDs have topographic height of $\sim 1.5 \mathrm{~nm}$. Consequently, most of the exfoliated GOQDs mainly exist as a single or few-layered graphene sheets.

(a)

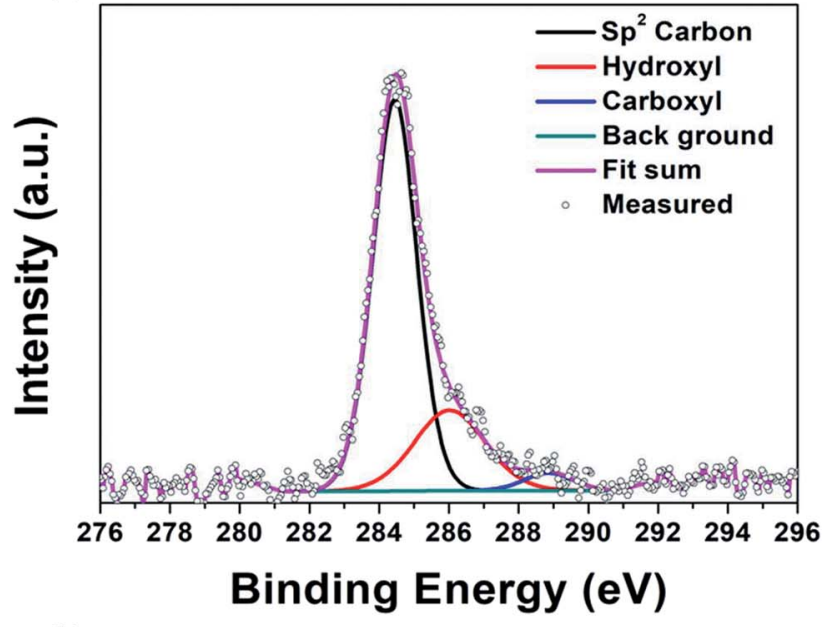

(b)

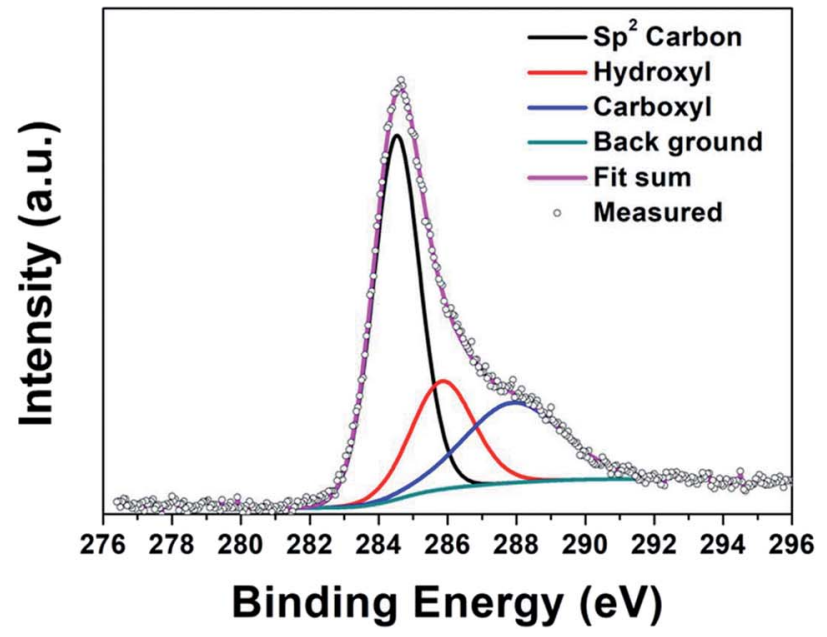

Fig. 3 XPS spectra GQDs and GOQDs. (a) GQDs and (b) GOQDs.
The size and height of GOQDs were comparable with those of the GQDs produced by low-energy laser source (i.e., longer wavelength). Also, the yield of PLAL process was calculated by dividing the weight of dried GQDs or GOQDs product by the weight of the starting MWCNTs. Based on this method, the yield of GQDs and GOQDs were determined as about $10-12 \%$.

The wavelength of laser can induce multiple effects on PLAL process. Noted that multiphoton absorption is a predominant ablation mechanism when high-frequency pulses such as nanosecond laser is employed for PLAL. The electron density $\left(n_{0}\right)$ induced by laser pulse depends on the photon energy $E$ and the number of photons $(N) .{ }^{\mathbf{2 1 , 2 8}}$ Laser pulse with high photon energy can lead to more significant multiphoton-ionization and Coulomb explosion, expediting decomposition of molecules. ${ }^{28-30}$ Hence, the solvent (i.e., ethanol in this study) can be photo-thermally decomposed during PLAL which forms cavitation bubbles, consisted of carbon- or oxygen-based small molecules, on the surface of MWCNTs. The surface functionalization of GQDs by oxygen functional groups during PLAL in ethanol can be described as eqn (1). ${ }^{31}$

$$
\begin{aligned}
& \mathrm{C}_{2} \mathrm{H}_{5} \mathrm{OH} \rightarrow \mathrm{C}_{2} \mathrm{H}_{4}+\mathrm{H}_{2} \mathrm{O} \\
& \mathrm{C}_{2} \mathrm{H}_{5} \mathrm{OH} \rightarrow \mathrm{CH}_{3}+\mathrm{CH}_{2} \mathrm{OH} \\
& \mathrm{C}_{2} \mathrm{H}_{5} \mathrm{OH} \rightarrow \mathrm{C}_{2} \mathrm{H}_{5}+\mathrm{OH}
\end{aligned}
$$

The shorter laser wavelength with higher photon energies can facilitate decomposition of ethanol, correspondingly an easy formation of $\mathrm{OH}$ functional groups or defects on the surface of the ablated GQDs or MWCNTs. The OH-enriched solvent can efficiently functionalize the defect-enriched surface of GQDs, and thus the transformation from GOQs to GOQDs likely occurs.

The optical properties of GQDs and GOQDs were investigated using PL (photoluminescence) and PLE (photoluminescence excitation) measurements. Fig. 4a show the PL spectra of the colloidal solutions of GQDs and the GOQDs. The PL intensity of GQDs was about 1.5 times higher than that of GOQDs. The digital images of the GQDs show distinct blue emission (the inset in Fig. 4a, left digital image), while the GOQDs exhibits a mixed emission of blue and green (the inset in Fig. 4a, right digital image). Also, the GQDs shows the excitation independent PL properties at excitation wavelength with 300 to $400 \mathrm{~nm}$, in contrast the GOQDs have excitation dependent PL properties (Fig. S4 $\dagger$ ). The PLE spectra was investigated at various detection emission wavelengths of PL spectrum (Fig. 4b). Both GQDs and GOQDs exhibit the PLE peaks at about 260 and $360 \mathrm{~nm}$. However, the GOQDs have much broader PLE peak than the GQDs with emission dependent PLE properties. The characteristics of PL and PLE spectra reflect that emissions from the GQDs and GOQDs may have a different mechanism.

To investigate PL mechanisms of GQDs and GOQDs, we carried out UV-vis and time-resolved photoluminescence (TRPL) analysis (Fig. 5). The PLE spectra of GOQDs shows excitation dependent PL properties (Fig. S4b广), and also the UV-vis absorbance of GOQDs shows a broad absorption spectrum with a gradual change up to $700 \mathrm{~nm}$ (Fig. 5a). These properties of GOQDs are similar to previously reported PLE and UV-vis 
(a)

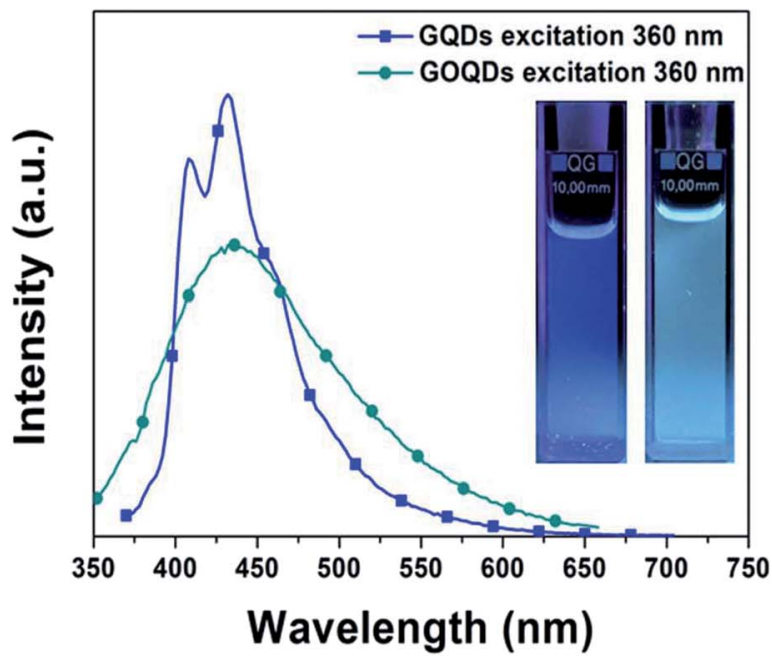

(b)

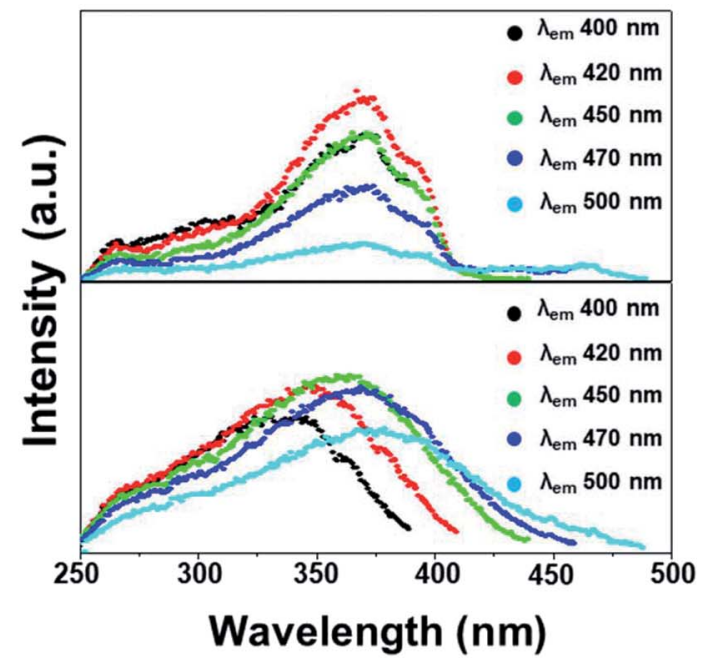

Fig. 4 (a) PL spectra of GQDs and GOQDs in ethanol. (b) PLE spectra of GQDs and GOQDs with various emission source.

(a)

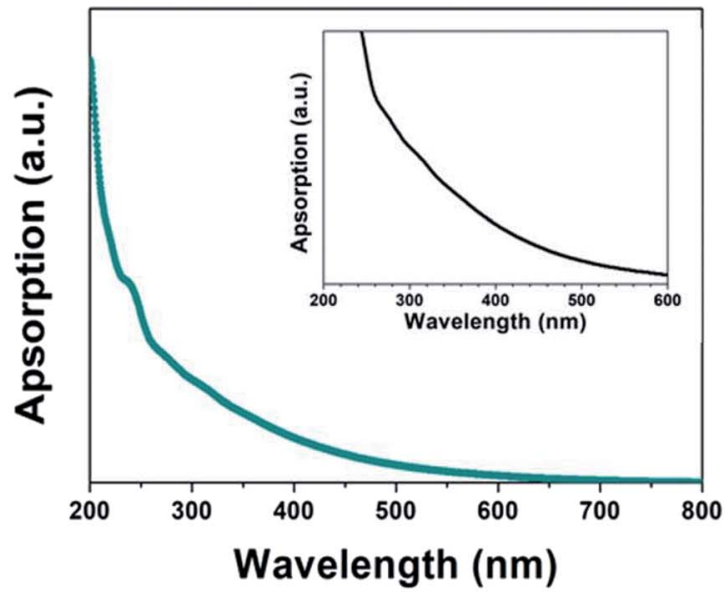

(c)

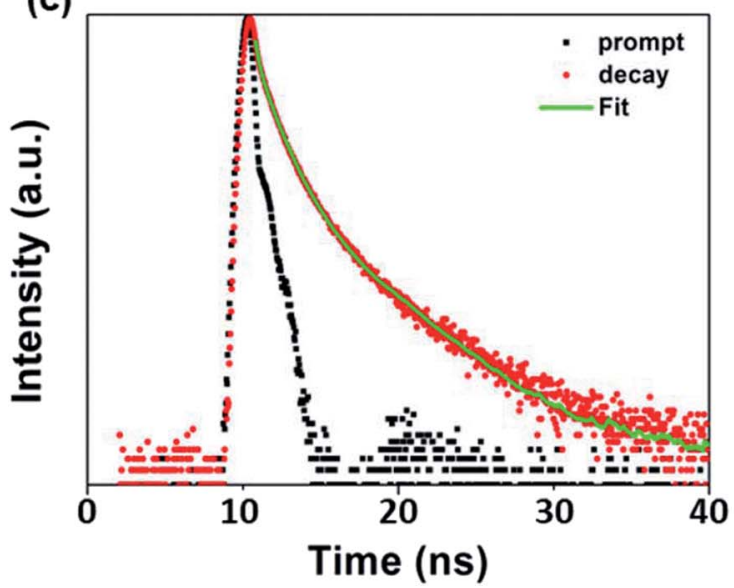

(b)

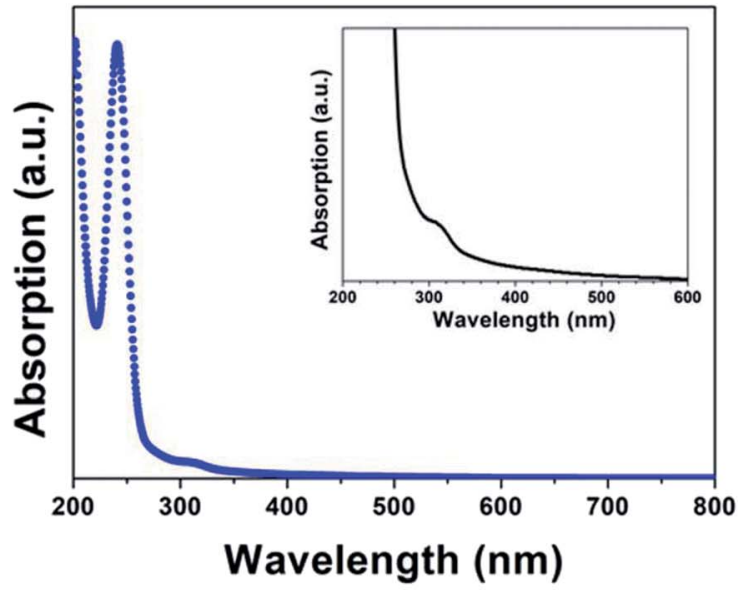

(d)

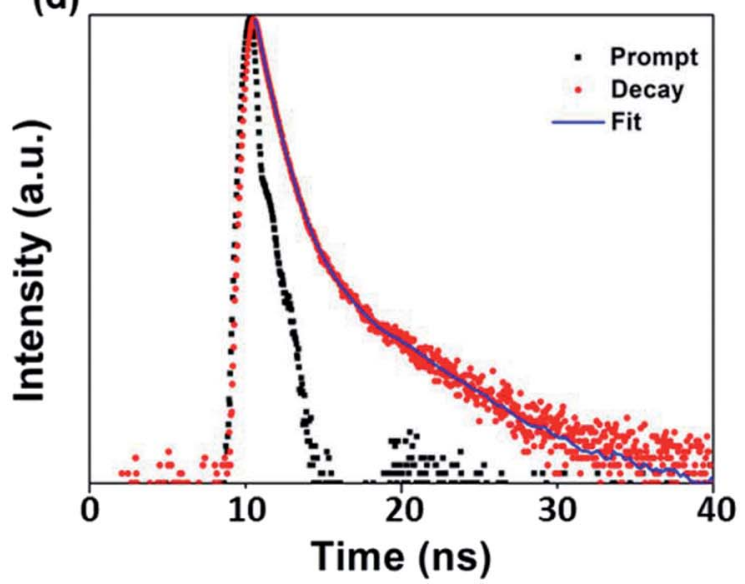

Fig. 5 (a, b) UV-vis spectra of GQDs and GOQDs, respectively. (c, d) TCSPC decay curves of the GQDs and GOQDs with excitation wavelength at $370 \mathrm{~nm}$ and emission wavelength at $450 \mathrm{~nm}$, respectively. The green and blue line show the three exponential fits of GQDs and GOQDs, respectively. 
absorbance results of GOQDs with oxygen-rich functional groups. ${ }^{32,33}$ Compared to GOQDs, the absorption spectrum of GQDs presents broad absorption peaks with high intensity at about 245 and $310 \mathrm{~nm}$, respectively (Fig. 5b). These absorption peaks correspond to PLE peaks at about 260 and $360 \mathrm{~nm}$, respectively (Fig. 4b). The mixed blue and green emission from GOQDs is originated from intrinsic states and/or extrinsic defect states in the bandgap. Notably, GQDs exhibits distinct blue emission with the strong absorption peak, indicating the intrinsic states in the band structure dominantly affect PL emissions of GQDs. ${ }^{2,7}$ To clarify possible recombination mechanisms of GQDs and GOQDs, we carried out TRPL analysis (Fig. 5c and d). Table 1 depicts the values obtained by timecorrelated single photon counting (TCSPC) characterization. The fluorescence decay curve is fitted with triexponential function (eqn (2)), where fluorescence decay occurs through three different relaxation pathways.

$$
\text { fit }=A+B_{1} \mathrm{e}^{(-t / \tau 1)}+B_{2} \mathrm{e}^{(-t / \tau 2)}+B_{3} \mathrm{e}^{(-t / \tau 3)}
$$

where " $\tau$ " is the fluorescence lifetime and " $B$ " represents amplitude of the corresponding lifetime. The obtained chisquare $\left(\chi^{2}\right)$ ranges between 1.05 and 1.1. The $\chi^{2}$ value in the range of $1.0<\chi^{2}<1.2$ is generally assumed to be acceptable for fitting. Generally, the emission that originates from defect states shows a longer recombination lifetime than that from intrinsic states. ${ }^{34-36}$ Also, among the three lifetimes, one is associated with an intrinsic state, while the other two are due to the existence of oxygen-rich functional groups on the surface of GQDs and GOQDs. Fluorescence lifetimes of GQDs and GOQDs are recorded at $450 \mathrm{~nm}$, where the excitation wavelength of a diode laser was $370 \mathrm{~nm}$. The lifetimes of GQDs are $\tau 1=1.3 \mathrm{~ns}$ (78\%), $\tau 2=3.1 \mathrm{~ns}(17 \%)$ and $\tau 3=11 \mathrm{~ns}(5 \%)$, whereas those of GOQDs are $\tau 1=0.7 \mathrm{~ns}(53 \%), \tau 2=3 \mathrm{~ns}(35 \%)$ and $9 \mathrm{~ns}(11 \%)$ (Table 1). Both of GQDs and GOQDs have a short-lifetime for $\tau 1$ (1.3 ns and $0.7 \mathrm{~ns}$ ), indicating that $\tau 1$ can be related to the emission from the intrinsic state. Hoffmann et. al. demonstrated that for a triplet ground state, the energy difference $(\delta E)$ between the $\sigma$ and $\pi$ orbitals should be below $1.5 \mathrm{eV} .{ }^{19}$ Two different electronic transitions were observed at $360 \mathrm{~nm}(3.4 \mathrm{eV})$ and $260 \mathrm{~nm}(4.76 \mathrm{eV})$ in the PLE spectra (Fig. $4 \mathrm{~b})$. Thus, $\delta E$ can be calculated as $1.36 \mathrm{eV}$ for GQDs and GOQDs, which is below the critical value of $1.5 \mathrm{eV}$. Hence, the assignment of two respective transition states is reasonable for GQDs and GOQDs. The blue emissions of GQDs can be attributed to the intrinsic state. The GQDs has a longer lifetime $(\tau 1)$ for intrinsic states than GOQDs, and therefore the green emission can be effectively suppressed (Fig. 4a). Compared to GQDs, the quantitative

Table 1 Excitation emission values, $\chi^{2}$ value, excitation lifetimes, and their corresponding amplitudes for GQDs and GOQDs

\begin{tabular}{llllll}
\hline & & & \multicolumn{1}{c}{$\begin{array}{l}\tau_{1} \\
(\mathrm{~ns}) / \beta_{1}\end{array}(\%)$} & $\begin{array}{l}\tau_{2} \\
(\mathrm{~ns}) / \beta_{2}\end{array}(\%)$ & $\begin{array}{l}\tau_{3} \\
(\mathrm{~ns}) / \beta_{3}(\%)\end{array}$ \\
\hline GQDs & $370 / 450$ & 1.05 & $1.3 / 78$ & $3.1 / 17$ & $11 / 5$ \\
GOQDs & $370 / 450$ & 1.1 & $0.7 / 53$ & $3 / 35$ & $9 / 11$
\end{tabular}

fraction of lifetimes for $\tau 2$ and $\tau 3$ was much higher for GOQDs (46\%) probably due to a large amount of oxygen-rich functional groups on the surface of GOQDs (Fig. 3b), resulting in mixed green and blue emission. The produced GQDs and GOQDs exhibit distinctly different optoelectronic properties, which can be used for different applications.

\section{Conclusions}

In conclusion, we successfully demonstrated that the GQDs and GOQDs with controllable oxygen functional groups can be easily prepared by simply changing the laser wavelength for PLAL. The oxygen-rich functional groups can be more easily derived from the solvent (i.e., ethanol) when the shorter wavelength of the laser pulses is employed to trigger photothermal decomposition of the ethanol. According to compositional and structural analysis, selective productions of GQDs and GOQDs can be achieved by modulating the laser wavelength for PLAL process. Chemical bonding of GQDs is mainly composed of pure $\mathrm{sp}^{2}$ carbons with weak oxygen groups, while the various oxygen-rich functional groups were presented on the surface of GOQDs. Furthermore, GQDs and GOQDs showed clearly different optical properties. GOQDs exhibited the blue to green emission, while the emission of GQDs is distinct blue emission. These result demonstrate that by changing the laser wavelength, the amount of oxygen-rich functional groups on the surface of GQDs or GOQDs can be controlled during PLAL process, resulting in modulated optoelectronic properties. Thus, our strategy is remarkably simple and facile way for selectively producing GQDs and GOQDs by PLAL process, which has a hudge potential to be applied in real optoelectonic applications such as optical devices or bioimaging.

\section{Conflicts of interest}

There are no conflicts to declare.

\section{Acknowledgements}

This work was supported by the Korea Institute of Industrial Technology as "Development of Additively Manufacturing Process Technology of Ni-based Superalloys for Ultra-high efficiency Power Plants" (KITECH EO-19-0016). This research was also supported by Basic Science Research Program through the National Research Foundation of Korea (NRF) funded by the Ministry of Science, ICT \& Future Planning 2018R1D1A1A02085938.

\section{Notes and references}

1 L. A. Ponomarenko, F. Schedin, M. I. Katsnelson, R. Yang, E. W. Hill, K. S. Novoselove and A. K. Geim, Science, 2008, 320, 356-358.

2 M. Bacon, S. J. Bradley and T. Nann, Part. Part. Syst. Charact., 2014, 31, 415-428.

3 L. Li, G. Wu, G. Yang, J. Peng, J. Zhao and J. J. Zhu, Nanoscale, 2013, 5, 4015-4039. 
4 S. Y. Lim, W. Shen and Z. Gao, Chem. Soc. Rev., 2015, 44, 362381.

5 A. Abbas, L. T. Mariana and A. N. Phan, Carbon, 2018, 140, 77-99.

6 S. Zhu, Y. Song, J. Wang, H. Wan, Y. Zhang, Y. Ning and B. Yang, Nano Today, 2017, 13, 10-14.

7 K. A. Fernando, S. Sahu, Y. Liu, W. K. Lewis, E. A. Guliants, A. Jafariyan, P. Wang, C. E. Bunker and Y. P. Sun, ACS Appl. Mater. Interfaces, 2015, 7, 8363-8376.

8 S. H. Song, M.-H. Jang, J. Chung, S. H. Jin, B. H. Kim, S.-H. Hur, S. Yoo, Y.-H. Cho and S. Jeon, Adv. Opt. Mater., 2014, 2, 1016-1023.

9 J. Shen, Y. Zhu, X. Yang and C. Li, Chem. Commun., 2012, 48, 3686-3699.

10 M. Zheng, S. Ruan, S. Liu, T. Sun, D. Qu, H. Zhao, Z. Xie, H. Gao, X. Jing and Z. Sun, ACS Nano, 2015, 9, 11455-11461.

11 Y. Shi, A. Pramanik, C. Tchounwou, F. Pedraza, R. A. Crouch, S. R. Chavva, A. Vangara, S. S. Sinha, S. Jones, D. Sardar, C. Hawker and P. C. Ray, ACS Appl. Mater. Interfaces, 2015, 7, 10935-10943.

12 J. Wen, Y. Xu, H. Li, A. Lu and S. Sun, Chem. Commun., 2015, 51, 11346-11358.

13 X. Tan, Y. Li, X. Li, S. Zhou, L. Fan and S. Yang, Chem. Commun., 2015, 51, 2544-2546.

14 M. Park, H. D. Ha, Y. T. Kim, J. H. Jung, S. H. Kim, D. H. Kim and T. S. Seo, Anal. Chem., 2015, 87, 10969-10975.

15 R. Liu, D. Wu, X. Feng and K. Mullen, J. Am. Chem. Soc., 2011, 133, 15221-15223.

16 H. Zhu, X. Wang, Y. Li, Z. Wang, F. Yang and X. Yang, Chem. Commun., 2009, 5118-5120.

17 Z. Li, W. Zhang, Y. Luo, J. Yang and J. G. Hou, J. Am. Chem. Soc., 2009, 131, 6320-6321.

18 Y. Li, Y. Hu, Y. Zhao, G. Shi, L. Deng, Y. Hou and L. Qu, Adv. Mater., 2011, 23, 776-780.

19 R. Hoffmann, J. Am. Chem. Soc., 1968, 90, 1475.
20 J. Yang, T. Ling, W. T. Wu, H. Liu, M. R. Gao, C. Ling, L. Li and X. W. Du, Nat. Commun., 2013, 4, 1695.

21 D. Zhang, B. Gokce and S. Barcikowski, Chem. Rev., 2017, 117, 3990-4103.

22 Z. Yan and D. B. Chrisey, J. Photochem. Photobiol., C, 2012, 13, 204-223.

23 V. Amendola and M. Meneghetti, Phys. Chem. Chem. Phys., 2013, 15, 3027-3046.

24 S.-L. Hu, K.-Y. Niu, J. Sun, J. Yang, N.-Q. Zhao and X.-W. Du, J. Mater. Chem., 2009, 19, 484-488.

25 S. H. Kang, S. Mhin, H. Han, K. M. Kim, J. L. Jones, J. H. Ryu, J. S. Kang, S. H. Kim and K. B. Shim, Sci. Rep., 2016, 6, 38423.

26 L. Wang, Y. Wang, T. Xu, H. Liao, C. Yao, Y. Liu, Z. Li, Z. Chen, D. Pan, L. Sun and M. Wu, Nat. Commun., 2014, $5,5357$.

27 L. Lin and S. Zhang, Chem. Commun., 2012, 48, 10177-10179. 28 E. Arola, IEEE J. Quantum Electron., 2014, 50, 1-12.

29 R. García-Calzada, M. Rodio, K. Bagga, R. Intartaglia, P. Bianchini, V. S. Chirvony and J. P. Martínez-Pastor, RSC Adv., 2015, 5, 50604-50610.

30 E. Boulais, R. Lachaine and M. Meunier, Nano Lett., 2012, 12, 4763-4769.

31 R. Sivaramakrishnan, M. C. Su, J. V. Michael, S. J. Klippenstein, L. B. Harding and B. Ruscic, J. Phys. Chem. A, 2010, 114, 9425-9439.

32 Z. Gan, H. Xu and Y. Hao, Nanoscale, 2016, 8, 7794-7807.

33 Z. Gan, S. Xiong, X. Wu, T. Xu, X. Zhu, X. Gan, J. Guo, J. Shen, L. Sun and P. K. Chu, Adv. Opt. Mater., 2013, 1, 926-932.

34 S. Ahirwar, S. Mallick and D. Bahadur, ACS Omega, 2017, 2, 8343-8353.

35 F. Liu, M. H. Jang, H. D. Ha, J. H. Kim, Y. H. Cho and T. S. Seo, Adv. Mater., 2013, 25, 3657-3662.

36 S. Zhu, J. Shao, Y. Song, X. Zhao, J. Du, L. Wang, H. Wang, K. Zhang, J. Zhang and B. Yang, Nanoscale, 2015, 7, 79277933. 